\title{
ORIGINAL ARTICLE Prognostic signature and clonality pattern of recurrently mutated genes in inactive chronic lymphocytic leukemia
}

AM Hurtado ${ }^{1}$, T-H Chen-Liang ${ }^{1}$, B Przychodzen ${ }^{2}$, C Hamedi $^{1}, \mathrm{~J} \mathrm{Juñoz-Ballester}^{1}$, B Dienes ${ }^{2}$, MD García-Malo ${ }^{1}$, Al Antón ${ }^{1}$, F de Arriba ${ }^{1}$, R Teruel-Montoya ${ }^{1}$, FJ Ortuño ${ }^{1}$, V Vicente ${ }^{1}$, JP Maciejewski ${ }^{2}$ and A Jerez ${ }^{1}$

An increasing numbers of patients are being diagnosed with asymptomatic early-stage chronic lymphocytic leukemia (CLL), with no treatment indication at baseline. We applied a high-throughput deep-targeted analysis, especially designed for covering widely TP53 and ATM genes, in 180 patients with inactive disease at diagnosis, to test the independent prognostic value of CLL somatic recurrent mutations. We found that 40/180 patients harbored at least one acquired variant with ATM $(n=17,9.4 \%)$, NOTCH1 $(n=14,7.7 \%)$, TP53 $(n=14,7.7 \%)$ and SF3B1 $(n=10,5.5 \%)$ as most prevalent mutated genes. Harboring one 'sub-Sanger' TP53 mutation granted an independent 3.5-fold increase of probability of needing treatment. Those patients with a double-hit ATM lesion (mutation+11q deletion) had the shorter median time to first treatment (17 months). We found that a genomic variable: TP53 mutations, most of them under the sensitivity of conventional techniques; a cell phenotypic factor: CD38-positive expression; and a classical marker as $\beta 2$-microglobulin, remained as the unique independent predictors of outcome. The high-throughput determination of TP53 status, particularly in this set of patients frequently lacking high-risk chromosomal aberrations, emerges as a key step, not only for prediction modeling, but also for exploring mutation-specific therapeutic approaches and minimal residual disease monitoring.

Blood Cancer Journal (2015) 5, e342; doi:10.1038/bcj.2015.65; published online 28 August 2015

\section{INTRODUCTION}

Nowadays, an increasing numbers of patients are being diagnosed with early-stage chronic lymphocytic leukemia (CLL), likely owing to the use of routine blood tests for health screening and the widespread availability of flow cytometry. ${ }^{1-3}$ Among this subset of CLL patients, most with a non-active disease and no treatment indication at baseline, different prognostic modeling approaches, incorporating traditional (clinical and laboratory), cytogenetic, immunophenotypic and immunoglobulin heavy-chain variable region gen $(\mathrm{IgVH})$ status, have been proposed. ${ }^{4,5}$

The contemporary vision of neoplasm development is based on a consecutive acquisition of genetic changes with a selection and expansion of the more fit population. ${ }^{6,7}$ The heterogeneous course of CLL is, to a certain extent, driven by the diverse combinations of clones with acquired chromosomal lesions and somatic mutations. ${ }^{8,9}$ Whole-exome studies (WES) have shown that the number of somatic variants per case is lower in CLL than that of those described in solid tumors and other leukemias, and that the set of genes affected is discreet. ${ }^{10-12}$ These two aspects make this disease a suitable candidate for deep-targeted sequencing, a technique focused on distinct genomic sites, which also enables reliable detection of subclonal mutations because of to its higher depth of coverage compared with WES. Using this more affordable and rapid strategy, recent studies have determined the prognostic impact of TP53 subclones. ${ }^{13}$ In addition, the status of ATM, NOTCH1 and SF3B1, also recurrently mutated in CLL, have been associated with impaired overall and treatment-free survival. ${ }^{14-16}$ Nevertheless, a comprehensive high-throughput sequencing study of these variants, assessing their clinical relevance, in the context of both traditional and newer factors, is lacking.
In this study, we propose to refine and apply a method for highthroughput targeted analysis of somatic recurrent mutations in CLL, especially designed for covering widely TP53 and ATM genes. Our main aim is to assess the independency of the prognostic value of those variants, related to time to first treatment and survival, in patients with CLL and no indication for therapy at diagnosis.

\section{MATERIALS AND METHODS}

\section{Patients}

From 2006 to 2012, presentation bone marrow aspirates or blood samples DNA was collected during the diagnostic workout from 265 consecutive CLL patients, after informed consent, according to the protocols approved by the Institutional Review Board of Hospital Morales Meseguer (EST-32/13) and with the Declaration of Helsinki. Patients who met criteria for an active disease at baseline, did not reach a minimum treatment-free follow-up of 3 months, or nucleic acids did not pass the quality control for either $\operatorname{lgVH}$ status or targeted sequencing, were excluded (Figure 1). Diagnosis and definition of active disease, requiring therapy, were achieved according to the International Workshop on Chronic Lymphocytic Leukemia established criteria. ${ }^{17}$ Time-to-first-treatment (TTFT) was measured from diagnosis to date of first treatment. Regular follow-up consisted of blood cell counts and clinical examinations every 3 months the first year after diagnosis, and henceforth, visits were carried out from 3 to 6 months, depending on patient risk.

\section{Diagnostic workout}

Every patient underwent a flow cytometry characterization with a panel including CD45, pan B-cell markers (CD19, CD20, CD22, CD79b, and surface immunoglobulin light chains), markers for differential diagnosis with other

\footnotetext{
${ }^{1}$ Hematology and Medical Oncology Department, Hospital Morales Meseguer, IMIB, Murcia, Spain and ${ }^{2}$ Traslational Hematology and Oncology Research, Cleveland Clinic, Cleveland, OH, USA. Correspondence: Dr A Jerez, Hematology and Medical Oncology Department, Hospital Morales Meseguer, IMIB, Marques de los Velez s/n, Murcia E-30008, Spain. E-mail: andjercay@gmail.com 
B-cell chronic lymphoproliferative diseases (CD5, CD23, FMC7, CD10, CD81, CD103, CD25 and CD11c) and prognosis markers (CD38 and ZAP70) (Antibodies from BD Biosciences, San Jose, CA, USA).

Fluorescence in situ hybridization (FISH) analysis was performed on interphase nuclei at diagnosis from directly harvested peripheral blood or bone marrow samples according to the manufacturer's protocol and using the following commercially available probes (Abbott Molecular, Des Plaines, IL, USA): LSI MYB (6q23), LSI P53 (17p13.1)/ LSI ATM (11q22.3), LSI D13S319 (13q14.3)/CEP12, as reported. ${ }^{18}$ A minimum of 400 nuclei were scored for each probe or probe combination.

Immunoglobulin heavy-chain variable diversity (D)-joining $(J)$ rearrangements were amplified from either reverse-transcribed total RNA (preferred source) or genomic DNA. Purified amplicons were sequenced either directly or on subcloning. ${ }^{19}$ Sequences were aligned to the ImMunoGeneTics for computation of mutational load. ${ }^{21}$ Sequences were considered mutated or not using the cutoff of $2 \%$ mismatch. ${ }^{22}$

\section{Targeted sequencing}

We designed a TruSeq Custom Amplicon panel (Illumina, Inc. San Diego, CA, USA) containing 13 genes and covering 28.099 bases (Table 1). For some genes known mutation hotspots were targeted; and for those with a widespread localization of the lesions, the entire coding sequence was analyzed. The average amplicon size was 238 base pairs and $~ 99.1 \%$ of the regions were covered on both strands. Library preparation was performed according to manufacturer's instruction. Paired-end sequencing

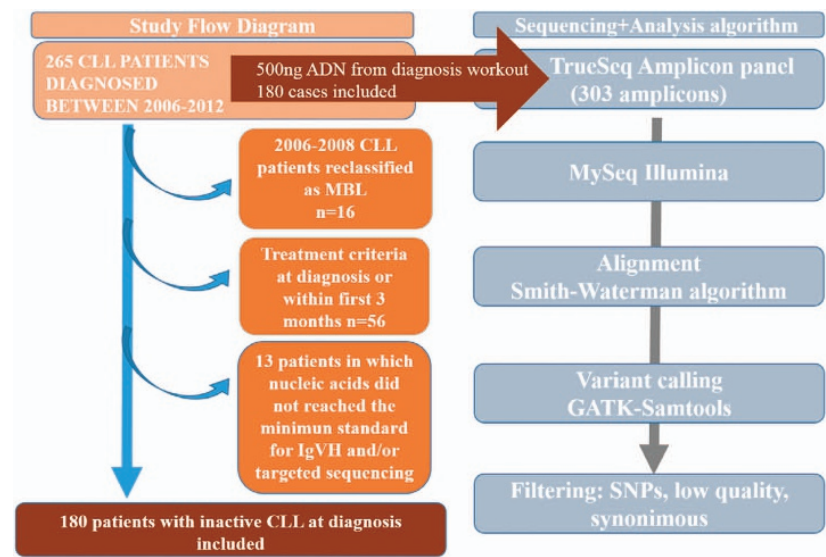

Figure 1. Study flow diagram. Visual representation of the exclusion criteria (left) and the targeted sequencing process pipeline (right).
( $2 \times 250 \mathrm{bp}$ ) was performed with MiSeq v2.2 chemistry, and a mean depth of $938 \mathrm{reads} / \mathrm{base}$ within the regions of interest was obtained. Raw data were analyzed with IlluminaonJboard Real Time Analysis (RTA v.2.4.60.8) software and MiSeq Reporter.

Variant call requirements and validation

The following conditions were established for a variant to be called: (i) to be non-synonymous; (ii) not to be listed in dbSNP database (NCBI Human Build 141); and (iii) a cutoff for any nucleotide position of 30 or more variant reads and a $\mathrm{Q}$ score $>30$ (see Variant call requirements: technique accuracy in Results). The filtered variant lists were manually reviewed and BAM file examined in Integrated Genome Viewer (Broad Institute).

Every variant with a clonal size of, at least, $20 \%$ and $>30$ variant reads were bi-directionally sequenced using an ABI 3730 DNA Analyzer (Life Technologies, Carlsbad, CA, USA).

Six TP53-mutated cases were selected for applying the whole-amplicon panel on germline DNA (four cases from CD3+ sorted cells, two cases from buccal mucosal swab) to test its somatic nature.

\section{Statistical analysis}

Comparisons of proportions and ranks of variables between groups were performed by $X^{2}$-test, Fisher's exact test, $t$-test or Mann-Whitney U-test, as appropriate. We used the Kaplan-Meier and the Cox method to analyse overall survival $(\mathrm{OS})$ and progression-free survival, with a two-sided $P$ value $\leqslant 0.05$ considered to be significant. In Cox models, examination of $\log (-\log )$ survival plots and partial residuals was performed to assess that the underlying assumption of proportional hazards was met. Thresholds of $<2.4 \mathrm{mg} / \mathrm{dl}$ for $\beta 2$-microglobulin, an absolute B-cell count of $11 \times 10^{9} / \mathrm{l}$ or over, and higher LDH levels than the upper normal limit ( $>$ UNL (that is, $378 \mathrm{U} / \mathrm{l})$, were chosen as reported elsewhere. ${ }^{23-25}$

\section{RESULTS}

Testing technique accuracy by resequencing

To estimate the accuracy of the technique to reproduce a variant call, 221 variants found in the first run were resequenced in depth (average reads per variant $\times 583$ ). Eighteen of these variants had a clonal size over Sanger sensitivity (>20\%), 58 variants with a clonal size between 10 and 20\%, and 145 variants below $10 \%$ of clone size. For this experiment, new libraries were built to capture and amplify exclusively the amplicons covering the variants selected, and the same genomic DNA used in the first run was used. Receiver operating characteristic curves for both number of variant reads and variant allelic frequency (VAF) were created by plotting the true positive rate (sensitivity) against the false-positive

Table 1. Targeted NGS panel characteristics

\begin{tabular}{|c|c|c|c|c|}
\hline Target gen & $\begin{array}{l}\text { Length covered } \\
\quad(b p)\end{array}$ & $\begin{array}{l}\% \text { Gen exome } \\
\text { sequenced }\end{array}$ & $\begin{array}{l}\% \text { CLL mutations } \\
\text { covered }^{*}\end{array}$ & Hotspot references \\
\hline TP53 & 2724 & $100 \%$ & $100 \%$ & \\
\hline ATM & 7411 & $56 \%$ & $78 \%(28 / 36)$ & Wang et $a l . .^{12}$ Puente et al..$^{10}$ \\
\hline $\mathrm{NOTCH} 1$ & 1487 & $19 \%$ & $100 \%(75 / 75)$ & Wang et al., ${ }^{12}$ Puente et al. ${ }^{10}$ \\
\hline SF3B1 & 3915 & $14 \%$ & $92 \%(22 / 24)$ & Wang et al. ${ }^{12}$ Puente et al. ${ }^{10}$ \\
\hline MYD88 & 296 & $31 \%$ & $100 \%(11 / 11)$ & Wang et al., 12 Puente et al. ${ }^{10}$ \\
\hline POT1 & 549 & $28 \%$ & $100 \%(5 / 5)$ & Wang et al., ${ }^{12}$ Puente et al. ${ }^{10}$ \\
\hline BIRC3 & 963 & $53 \%$ & $100 \% /(11 / 11)$ & Wang et al., ${ }^{12}$ Puente et al., ${ }^{10}$ Rossi et al..$^{45}$ \\
\hline KRAS & 567 & $100 \%$ & $100 \%$ & \\
\hline NRAS & 570 & $100 \%$ & $100 \%$ & \\
\hline U2AF1 & 572 & $100 \%$ & $100 \%$ & \\
\hline$B R A F$ & 357 & $14 \%$ & $100 \%(2 / 2)$ & $\begin{array}{l}\text { Wang et al., }{ }^{12} \text { Puente et al., }{ }^{10} \\
\text { Jebaraj et al. }{ }^{46}\end{array}$ \\
\hline$B C O R$ & 5268 & $100 \%$ & $100 \%$ & Wang et al., ${ }^{12}$ Puente et al. ${ }^{10}$ \\
\hline SETBP1 & 3420 & $71 \%$ & $100 \%(2 / 2)$ & Wang et al. ${ }^{12}$ Puente et al. ${ }^{10}$ \\
\hline TOTAL & 28099 & & & \\
\hline
\end{tabular}

Abbreviations: $\mathrm{bp}$, base pair; $\mathrm{CLL}$, chronic lymphocytic leukemia. Genes included, total gene length covered, percentage of gene exon sequenced and percentage of CLL mutations covered using as reference those variants described in the whole-exome studies referred in the last column. 
rate (1-specificity) at various threshold settings. The number of variant reads in the initial run showed to be more accurate to predict the reproducibility of the variant in a second run, with an area under the curve of $0.894(P \leqslant 0.001 ; 95 \% \mathrm{Cl}, 0.817-0.970)$, than the clonal size (VAF) (area under the curve: 0.613; $P=0.037 ; 95 \%$ $\mathrm{Cl}, 0.487-0.738$; Supplementary Figures $1 \mathrm{~A}$ and $1 \mathrm{~B})$. A cutoff of 30 reads was chosen as threshold to consider a variant reproducible with a sensibility of 0.85 and a specificity of 0.945 . No discrepancy was found when Sanger sequencing 15 selected variants over $20 \%$ of clonal size. None of the 13 variants harbored by six TP53-mutated cases was called when applying the panel on germline DNA.

Cohort and distribution of mutations. Two hundred and sixty-five patients were diagnosed in our center of a CLL from 2006 to 2012. Patients who, without needing therapy, did not achieve a minimum of 3 months of follow-up, were not contemplated (Figure 1). No monoclonal lymphocytosis cases were considered. The baseline characteristics of the 180 patients finally included were in accordance with their indolent-no need for treatment status at diagnosis, with $93 \%$ of the cohort assigned to Rai Stages 0 and I (Table 2).

We found that $40 / 180(22.2 \%)$ patients harbored at least one mutation; ATM $(n=17,9.4 \%)$, NOTCH1 $(n=14,7.7 \%)$, TP53 $(n=14$, $7.7 \%)$, SF3B1 $(n=10,5.5 \%), B C O R(n=3,1.6 \%), B I R C 3(n=2,1.1 \%)$, KRAS $(n=2,1.1 \%)$, U2AF1 $(n=2,1.1 \%)$, POT1 $(n=2,1.1 \%)$, MYD88 $(n=1,0.6 \%)$, and SETBP1 $(n=1,0.6 \%)$. No somatic variants were identified for BRAF and NRAS (Figure 2). Sixty-eight mutations were detected in the whole cohort with 18 deletions causing a frameshift, 1 non-frameshift deletion, 1 non-frameshift insertion and 48 missense single-nucleotide variants. Forty-one out of 68 mutations were already reported to the Catalog of Somatic Mutations in Cancer (COSMIC; http://cancer.sanger.ac. uk/cancergenome/projects/cosmic), as human cancers variants (Supplementary Table 1).
SF3B1 and NOTCH1 mutations were mutually exclusive, and a significant correlation between NOTCH1 mutations and the presence of a trisomy 12 was found $(P<0.01)$.

Clonal diversity of recurrently mutated genes in early-stage CLL. As samples used for this sequencing study belong to the diagnostic immunophenotypic workout, we could estimate the clonal or subclonal nature of the acquired mutations, adjusting the variant allele burden in non-sorted blood or bone marrow DNA for the percentage of the immunophenotypically quantified CLL population. In eight patients with a del11q or del 17p and a ATM or TP53 mutation, VAF was adjusted considering the loss of heterozygosity (Figure 3).

Thirty-five mutations were estimated to be clonal, that is, present in the whole tumor population, either in a heterozygous $(n=28)$ or hemizygous $(n=7)$ configuration. The allelic ratio of other mutations showed that they appeared only in a fraction of tumor cells, indicating that they were secondarily acquired or subclonal $(n=33)$. Certain genes showed predominantly clonal mutations ( $71 \%$ of NOTCH1, $71 \%$ of ATM variants), whereas others were mainly subclonal ( $80 \%$ of TP53 variants, $100 \%$ of Ras-family and U2AF1 genes).

Clinical correlates. When confronting the 40 patients with, at least, one mutation, with the 140 non-mutated patients, we found less patients stratified as stage 0 in the mutated group, in favor of stages I and II. Focusing on FISH abnormalities, both $13 q$ and +12 shown to be more frequent in mutated cases, though none of these differences reached the statistical significance (Table 2).

Likewise, no significant disparity was found when considering leukocyte, lymphocyte and platelet counts or hemoglobin, lactate dehydrogenase and $\beta_{2}$-microglobulin levels. In addition, no specific CD38 or ZAP70 expression pattern was observed to be characteristic of each group.

Table 2. Characteristics of patients included in the study at baseline and according to the presence or absence of, at least, a mutation

\begin{tabular}{|c|c|c|c|c|}
\hline Variable & Total $(\mathrm{n}=180)$ & (A) Mutated cases $(n=40)$ & (B) Non-mutated cases $(n=140)$ & $\mathrm{P}(A$ vs $B)$ \\
\hline Age, years, (mean \pm s.d.) & $69 \pm 1$ & $70 \pm 12$ & $68 \pm 11$ & 0.9 \\
\hline Sex, male/female, $\%(n)$ & $59(107) / 41(74)$ & $67(27) / 33(13)$ & $60(85) / 40(55)$ & 0.3 \\
\hline \multicolumn{5}{|l|}{ Rai Stage, \% } \\
\hline 0 & 45 & 38 & 49 & 0.3 \\
\hline I & 48 & 52 & 47 & 0.5 \\
\hline ॥ & 7 & 10 & 4 & 0.3 \\
\hline Leukocytes, x109/I (median, IQR) & $15.3(10.9-26.9)$ & $16.6(10.9-24.2)$ & $14.6(10.7-24)$ & 0.2 \\
\hline Lymphocytes, x $10^{9} /$ I (median, IQR) & $10.3(7-19.8)$ & $12.2(7.3-20.6)$ & $9.8(7-16)$ & 0.3 \\
\hline B-cell count, $\times 10^{9} / \mathrm{I}$ (median, IQR) & $9.1(6.1-17.3)$ & $11.2(7.4-19.1)$ & $8.9(6-15.6)$ & 0.3 \\
\hline Hemoglobin, g/dl (median, IQR) & $13.8(12.2-14.7)$ & $13.7(12-14.5)$ & $13.8(12.3-14.7)$ & 0.6 \\
\hline Platelets, $\times 10^{9} / \mathrm{I}$ (median, IQR) & $182(136-217)$ & $168(126-216)$ & $187(136-217)$ & 0.6 \\
\hline LDH IU/I (median, IQR) & $368(323-425)$ & $377(318-453)$ & $324(288-390)$ & 0.4 \\
\hline $\mathrm{B}_{2}$ microglobulin, mg/l (median, IQR) & $2.2(1.8-3)$ & $2.4(1.8-3.6)$ & $2.2(1.7-3)$ & 0.3 \\
\hline ZAP70 +, \% (n) & $42(76)$ & $50(20)$ & $40(56)$ & 0.1 \\
\hline CD38 +, \% (n) & $28(50)$ & $38(15)$ & $22(30)$ & 0.1 \\
\hline IgHV-unmutated status, \% ( $n$ ) & $34(61)$ & $43(17)$ & $27(37)$ & 0.07 \\
\hline \multicolumn{5}{|l|}{ FISH abnormalities, \% (n) } \\
\hline $\operatorname{Del}(13 q)$ & $46(84)$ & $50(20)$ & $47(65)$ & 0.7 \\
\hline+12 & $18(32)$ & $25(10)$ & $14(20)$ & 0.2 \\
\hline $\operatorname{Del}(11 q)$ & $5(8)$ & $17(7)$ & $1(0.7)$ & 0.01 \\
\hline $\operatorname{Del}(17 p)$ & $2(4)$ & $10(4)$ & $2(1.4)$ & 0.02 \\
\hline
\end{tabular}

Abbreviations: CD38, cluster of differentiation 38; FISH, fluorescence in situ hibridization; IgVH, immunoglobulin heavy-chain variable region gen; IQR, interquartile range; $L D H$, lactate dehydrogenase; ZAP70, Zeta-chain-associated protein kinase $70 ;+12$, trisomy 12 . Bold face: del(11q) and del (17p) as variables distributed differently among mutated or not mutated patients with statistical significance $(P=0.014)$. Note: quantitative variables with a normal distribution are expressed as mean \pm s.d. Quantitative variables not following the normal distribution are expressed as median and interquartile ranges. 


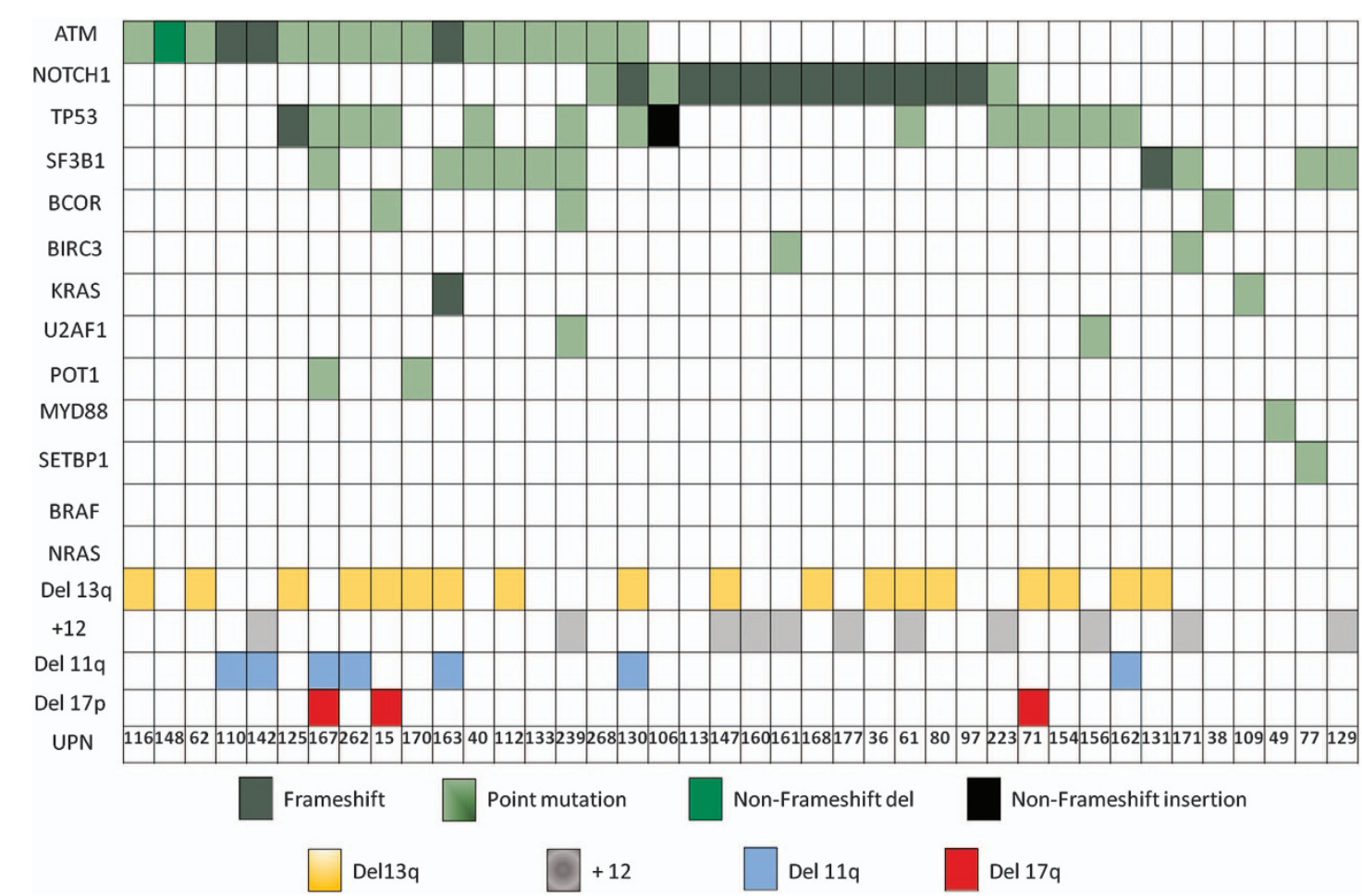

Figure 2. Distribution of mutations and chromosomal aberrations.

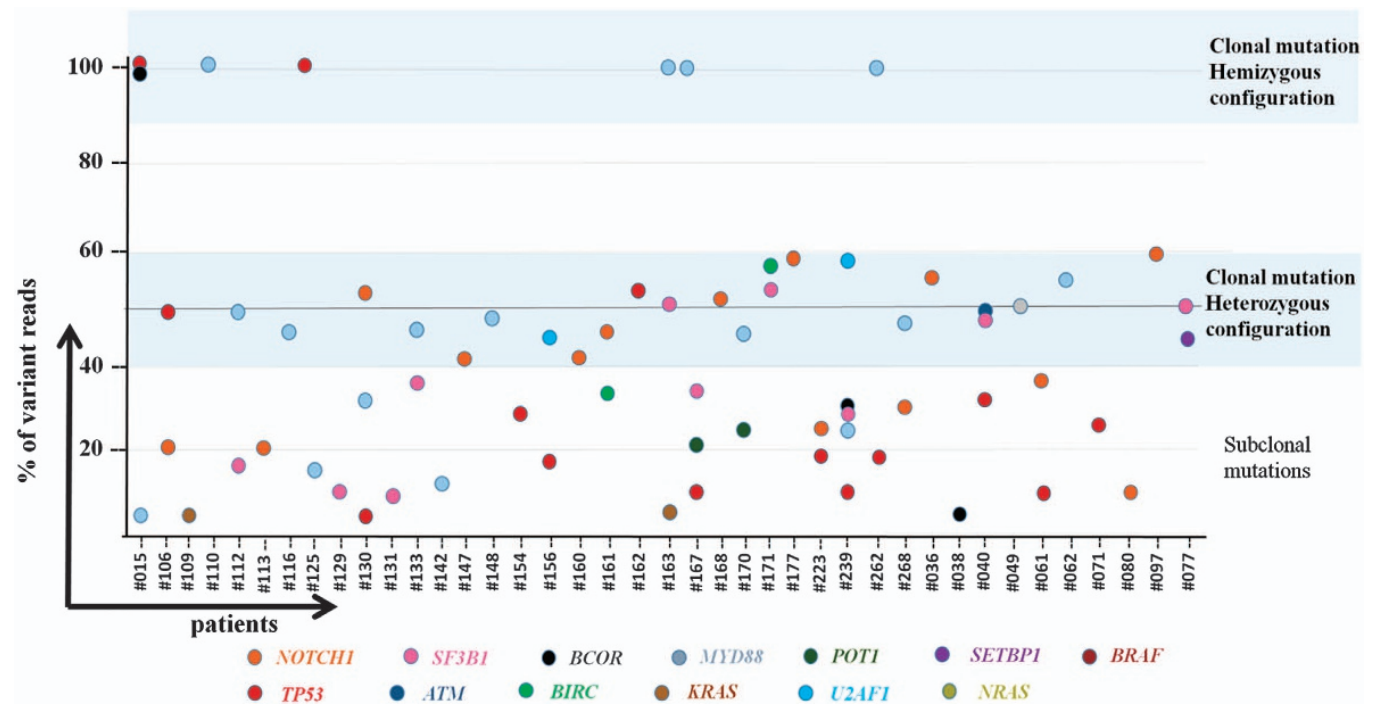

Figure 3. Estimated variant allele frequencies in the tumor fraction. The vertical axis represents allele frequency. Patients mutated are depicted in the abscissa. Color-coded circles for each gene affected. The variant allele frequency in non-sorted blood or bone marrow DNA was adjusted for the percentage of the immunophenotypically quantified CLL population.

Despite the low number of $17 p$ and $11 q$ deletions in our nonaggressive cohort, they were found mostly in the mutated group; the only difference statistically significant.

With a median follow-up of 54 months (interquartile range, 42-85 months), the median OS of the whole cohort has not been reached. Forty-two patients (23.3\%) required therapy. Median time to treatment for all patients has not been reached. Considering only those patients who were treated, median time to first treatment was 48 months (range, 5-96 months).

Patients with, at least, one mutation had a worse time to first treatment (median TTFT: 60 months vs not reached; $P \leqslant 0.001$; hazards ratio $(\mathrm{HR})=5.8 ; 95 \% \mathrm{Cl}, 3.1-10.9$; Figure $4 \mathrm{a})$ than those cases without a detected mutation, and a shorter median OS (54 months vs not reached; $P=0.01 ; \mathrm{HR}=3.9 ; 95 \% \mathrm{Cl}, 2.2-6.9$; Figure $4 \mathrm{f}$ ).

TP53-mutated cases showed both a shorter TTFT (median 29 months vs not reached; $P \leqslant 0.001 ; \mathrm{HR}=5.3 ; 95 \% \mathrm{Cl}, 2.6-10.8 \mathrm{Cl}$; Figure 4b) and OS (median 48 months; $P \leqslant 0.001 ; \mathrm{HR}=3.7 ; 95 \% \mathrm{Cl}$, 1.9-7.2; Figure 4g) than the wild-type cases (median not reached). The presence of a mutation in ATM predicted both for a shorter TTFT (mean 60 months; $P \leqslant 0.001 ; \mathrm{HR}=5 ; 95 \% \mathrm{Cl}, 2.5-10.2 \mathrm{Cl}$; Figure 4c) and OS (median 61 months; $P=0.016 ; \mathrm{HR}=2.5 ; 95 \% \mathrm{Cl}$, 1.2-5.2 Cl; Figure 4h). 
a

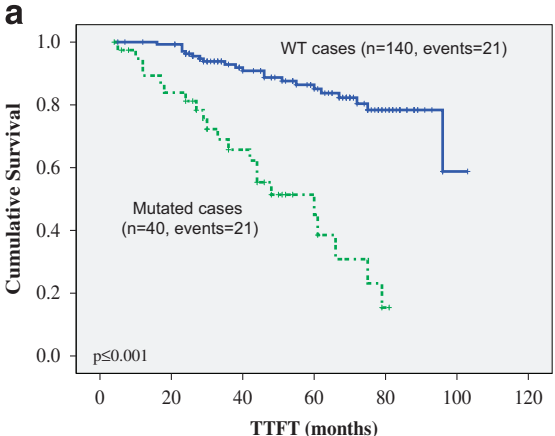

d

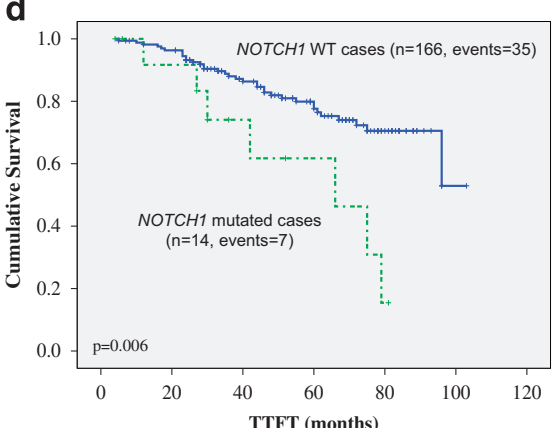

g

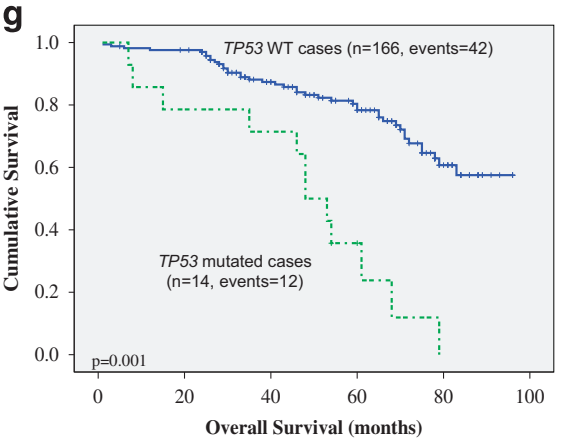

b

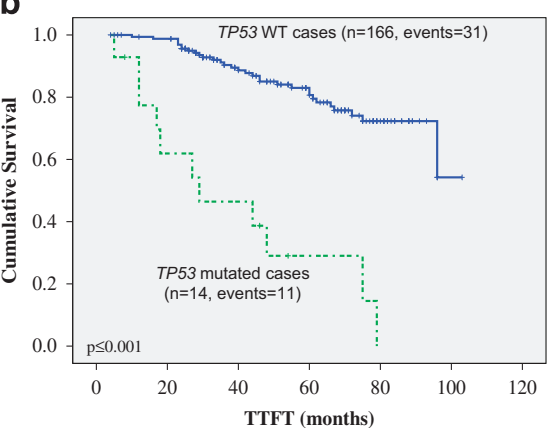

e

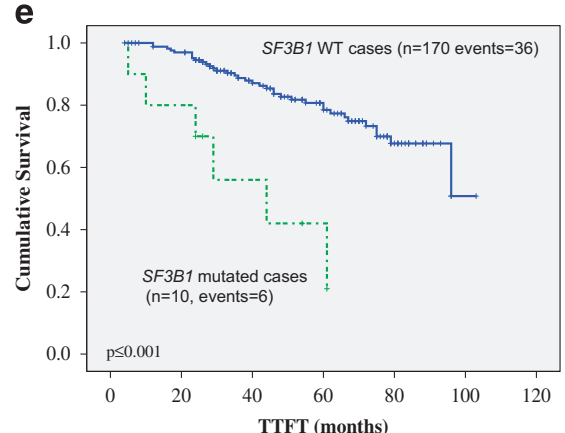

h

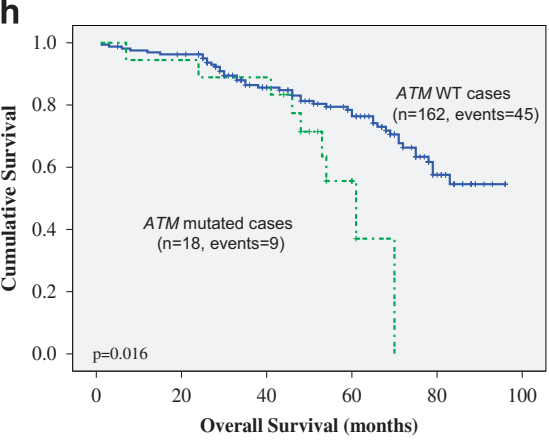

j

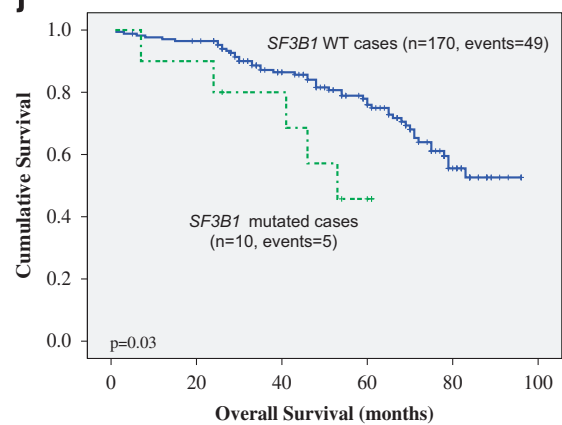

C

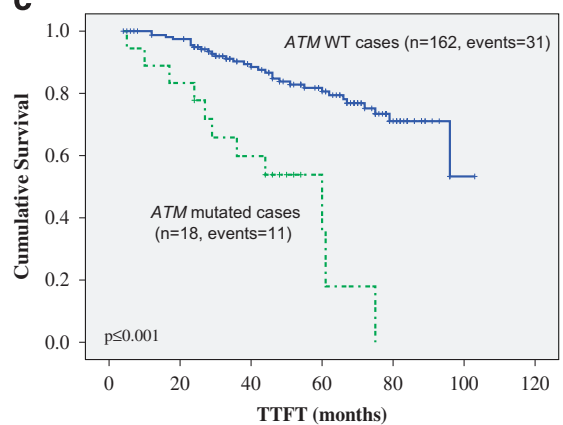

f

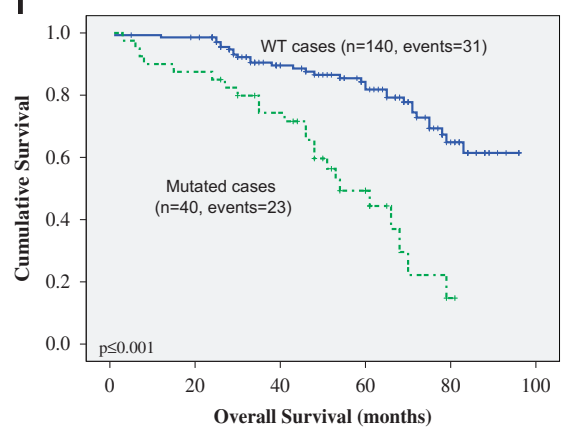

i

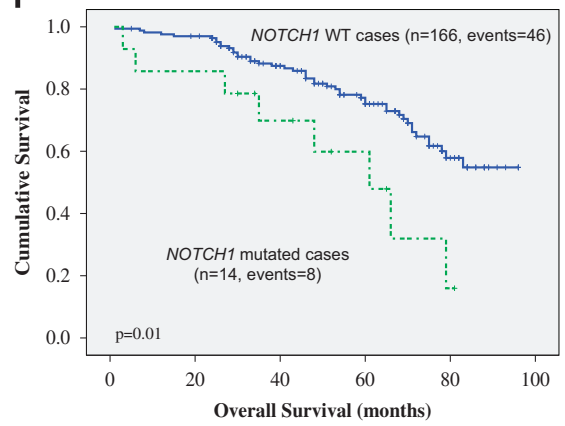

Figure 4. Differences in time to first treatment (TTFT) and survival outcomes (OS) in patients with, at least a mutation vs non-mutated (a, f); TP53 mutated or WT (b, g); ATM mutated or WT (c, h); NOTCH1 mutated or WT (d, i); and SF3B1 mutated or WT (e, j). P-values presented correspond to the Cox regression between the groups indicated.

NOTCH1-mutated cases also presented a decreased TTFT (66 months; $P=0.006 ; \mathrm{HR}=3.1 ; 95 \% \mathrm{Cl}, 1.4-7.1 ;$ Figure $4 \mathrm{~d}$ ) and a shorter median OS (61 months; $P=0.01 ; \mathrm{HR}=2.7 ; 95 \% \mathrm{Cl}, 1.3-5.7$; Figure 4i) than the wild-type cases (not reached). SF3B1-mutated patients also showed worse prognosis both in terms of TTFT (median 44 months; $P \leqslant 0.001 ; \mathrm{HR}=5.3,95 \% \mathrm{Cl}, 2.2-12$; Figure $4 \mathrm{e}$ ) and of $\mathrm{OS}(P=0.05 ; \mathrm{HR}=2.7 ; 95 \% \mathrm{Cl}, 1-7.8$; Figure $4 \mathrm{j})$ than the wild-type cases (mean not reached).

The low number of $\operatorname{del}(11 q)$ and $\operatorname{del}(17 p)$ cases, and the potential correlation of regressors with ATM and TP53 mutations (that is, variables strongly related to each other, measuring the same effect), precluded us from using them in this step of the analysis.

Mutational status of TP53, ATM, NOTCH1, SF3B1, IgVH status, the presence of +12 , del( $13 q)$, the Rai Stage, the positive expression of CD38 and ZAP70, $\beta 2$-microglobulin and LDH serum levels, and a B-cell count $\geqslant 11 \times 10^{9} / \mathrm{I}$ were evaluated in a univariate Cox regression, both for TTFT and OS (Supplementary Tables 2A and B). Only those variables with a $P \leqslant 0.05$ were included in a multivariate analysis (Tables $3 \mathrm{~A}$ and $\mathrm{B}$ ). The presence of a somatic 
Table 3A. Multivariate Cox regression for time to first treatment

\begin{tabular}{lcc}
\hline Variable & $R R(95 \% \mathrm{Cl})$ & P-value \\
\hline TP53 & $\mathbf{3 . 9}(\mathbf{1 . 4 - 1 0 . 9 )}$ & $\mathbf{0 . 0 1}$ \\
IgVH & $2(0.9-4.4)$ & 0.08 \\
NOTCH1 & $1.9(0.7-5.7)$ & 0.2 \\
ATM & $1.9(0.7-4.6)$ & 0.17 \\
+12 & $1.3(0.6-2.7)$ & 0.4 \\
CD38 & $\mathbf{2 . 3}(\mathbf{1 - 5 . 3 )}$ & $\mathbf{0 . 0 4}$ \\
SF3B1 & $2.6(0.8-8)$ & 0.09 \\
Rai Stage $>0$ & $1.1(0.5-2.3)$ & 0.7 \\
B-cell count $\geqslant 10 \times 10^{9} / 1$ & $1.9(0.9-3.9)$ & 0.08 \\
及2-microglobulin $\geqslant \mathbf{2 . 4}$ & $\mathbf{2 . 4}(\mathbf{1 . 2 - 4 . 8 )}$ & $\mathbf{0 . 0 2}$ \\
\hline
\end{tabular}

Abbreviations: CD38, cluster of differentiation 38; Cl, confidence interval; $\mathrm{IgVH}$, immunoglobulin heavy-chain variable region gen; $\mathrm{RR}$, relative risk; +12 , trisomy 12 . Bold face: variables reaching the independent statistical significance.

Table 3B. Overall survival

\begin{tabular}{lcc}
\hline Variable & $R R(95 \% \mathrm{Cl})$ & P-value \\
\hline TP53 & $\mathbf{2 . 5}(\mathbf{1 . 2 - 6 . 4 )}$ & $\mathbf{0 . 0 2}$ \\
IgVH & $1.5(0.8-2.6)$ & 0.2 \\
B2-microglobulin $\geqslant \mathbf{2 . 4}$ & $\mathbf{1 . 9}(\mathbf{1 . 1 - 3 . 4 )}$ & $\mathbf{0 . 0 4}$ \\
NOTCH1 & $1.8(0.7-4.5)$ & 0.2 \\
SF3B1 & $2.5(0.9-7.2)$ & 0.08 \\
Rai Stage $>0$ & $1.1(0.5-2.3)$ & 0.7 \\
ATM & $0.9(0.4-2.5)$ & 0.9 \\
\hline
\end{tabular}

Abbreviations: $\mathrm{CD} 38$, cluster of differentiation $38 ; \mathrm{Cl}$, confidence interval; $\mathrm{IgVH}$, immunoglobulin heavy-chain variable region gen; RR, relative risk; +12 , trisomy 12 . Bold face: variables reaching the independent statistical significance.

variant in TP53, a positive CD38 antigen expression and $\beta 2$ microglobulin serum levels above $2.4 \mathrm{mg} / \mathrm{dl}$, prevailed as independent variables linked to a shorter time for needing treatment, whereas only TP53 mutational status and $\beta 2$-microglobulin levels remained as significant predictors for OS.

We next sought to explore the impact of TP53 lesions below the sensitivity of Sanger. That is, to define the role of high-throughput sequencing in defining the prognosis in our cohort, as TP53 mutations remained as the only independent genomic variable. In this sub-analysis we excluded five patients with a TP53 lesion detectable by conventional techniques (direct sequencing and FISH/cytogenetics): one patient with a subclone harboring a TP53 mutation and a del(17p), two patients with a clone with both a TP53 and a del(17p), a case with a del(17p) in a clonal fashion but not detectable mutation, and one patient with a clonal TP53 mutation and $37 \%$ of variant reads. The other 10 subclonal cases would have been missed by Sanger sequencing as they called in $<20 \%$ reads (seven of them would have been missed even in B-cell-sorted DNA). We then replicated the multivariate Cox regression analyses shown in Tables $3 \mathrm{~A}$ and $\mathrm{B}$. Harboring one of these ten 'sub-Sanger' TP53 mutations granted an independent 3.5 -fold increase of probability of needing treatment during the course of the disease than a wild-type patient $(P=0.04 ; \mathrm{HR}=3.5$; $95 \% \mathrm{Cl}, 1-12.2)$, but it did not reach the significance for predicting OS $(P=0.15 ; \mathrm{HR}=2 ; 95 \% \mathrm{Cl}, 0.8-5.5)$.

Finally, a second sub-analysis showed that those patients with a double-hit ATM lesion (mutation+11q deletion) had the shorter median TTFT reported in this study (17 months) strikingly reduced compared with one-hit ATM patients (60 months). This impact was not seen in multivariate analysis or concerning OS (Supplementary Figure 2).

\section{DISCUSSION}

In the last few years a boost in the number of patients being diagnosed with early-stage CLL, not requiring therapy at diagnosis has been stated..$^{1-3}$ In this subset, we have shown that TP53 highthroughput mutational status emerges as an independent predictor, even when adjusting for the other recurrent gene variants in CLL, and for both traditional and recently reported prognostic factors. ${ }^{24}$ Surprisingly, even including newer genomic prognostic factors, a classical serologic parameter as $\beta 2$-microglobulin levels, remained an independent predictor both for TTFT and OS, whereas a positive CD38 expression also independently predicted a shorter TTFT.

The baseline characteristics of the patients included in our study were in accordance with their indolent-no need for treatment status. As other groups have shown, the new definition of CLL, excluding those cases with a B-monoclonal population of $<5000 /$ ul, determined in our cohort a shift toward higher Rai Stages and a higher rate of patients progressing and needing therapy. ${ }^{26,27}$

Patients with deletions on chromosome $17 p$ respond worse to treatment than do those without it, resulting in early relapse and shorter survival. ${ }^{28-30}$ This cytogenetic lesion can be found in up to $50 \%$ of relapsed and refractory patients, but is rare at baseline (5-10\%). ${ }^{8}$ We have found a $5 \%$ of TP53 mutations in a subset of patients where a 'wait and watch' therapeutical strategy is currently recommended. The relevant frequency and prognostic impact of TP53 variants represented in $<20 \%$ leukemic cells was recently reported by Rossi, et al. ${ }^{13}$ Of note, we show here that the majority of TP53 mutations, at baseline in our inactive CLL subset, were under the sensitivity of conventional sequencing, and that they kept its independent impact on prognosis even when adjusting by high-throughput detected ATM, NOTCH1 and SF3B1 variants. Given the markedly short TTFT and OS of our TP53mutated patients, it would seem an attractive approach to consider investigational studies directed to eradicate these subclones, in which these patients could be treated. Closely related, Farooqui et al. $^{31}$ reported a remarkable 2 -year survival and excellent drug-side-effects profile in the largest series of Ibrutinib therapy in treatment-naive patients with CLL and 17p deletions. In addition, identification of these subclones emerges as crucial as a concern is raised for clonal evolution and selection of resistant clones due the use of conventional (p53-dependent DNA damage) chemotherapeutic agents. ${ }^{9,32}$

The frequency of ATM mutations in large series of CLL patients it is not so well understood, mostly owing to the fact that its size and the scattered distribution of its somatic mutations precludes the drafting of an amplicon-limited design. ${ }^{33,34}$ We found ATM to be the most frequently mutated gene, with a predominance of clonal variants, indicating the ancestral-founding nature of these lesions. In addition, we found that the group with a shorter TTFT in our study was defined by those patients with a double-hit ATM. Likely, the reduced number of cases accounts for not reaching a statistical significance in multivariate or when addressing OS. Our finding supports the notion of a biological and clinical separation of double-hit ATM cases, from those where 11q deletion and the presence of an undamaged ATM allele give rise to a functional protein. $^{35-37}$

Recent studies have suggested an important prognostic role for NOTCH1 and SF3B1 in CLL. ${ }^{15,38,39}$ However, no study has performed a multivariate analysis including traditional clinical and laboratory markers, flow cytometry factors, lgVH status and, at least, the presence of ATM, TP53, NOTCH1 and SF3B1 variants. In addition, using high-throughput sequencing seems essential to discern the independent prognostic value of these mutations, as sub-Sanger mutations accounted for $25 \%$ of the variants in our study. Though strongly associated with a worse outcome and TTFT in univariate analysis, NOTCH1 and SF3B1 did not kept its significance when including TP53 and ATM mutations. Five out 
of seven, and four out of five NOTCH1 and SF3B1-mutated cases, which needed therapy, also harbored a TP53/del 17p and/or ATM/del11q lesion. This co-occurrence with more aggressive alterations can explain, in part, the lack of independent predictive value of SF3B1 and NOTCH1 in our cohort. Consistent with previous reports including aggressive disease cases, in our earlystage CLL cohort, SF3B1 and NOTCH1 were mutually exclusive and a correlation between NOTCH1 and trisomy 12 was found. ${ }^{40,41}$

Previous studies have indicated that the prognostic significance of $\mathrm{lgVH}$ status is independent from that of classical clinical stages, markedly in patients with early-stage disease. ${ }^{5,42}$ In our work, the inclusion of high-throughput mutational status ousted $\mathrm{IgVH}$ from the group of independent predictors, though it showed a trend for a shorter TTFT. ATM-mutated cases were found in a significant higher proportion among lgVH-unmutated cases. We did not find a different distribution of the IgVH status between TP53-mutated cases. These mutated cases can occur in both CLL IgVH subgroups. The late acquisition, autonomous of the emergence of the ancestral clone, of most TP53 mutations $(80 \%$ are subclones in our work), might justify this lack of association. Rossi et al. ${ }^{13}$ recently reported a similar frequency of $\mathrm{gVVH}$ non-mutated cases in TP53 subclonal and wild-type cases.

Ours is a translational study, with the focus shifted to clinical pragmatism. In that sense, the clonal or subclonal nature of the mutations is not as crucial as its detectability by Sanger. Given that most of our cohort patients were lacking high-risk chromosomal alterations, we aimed to define the clinical impact of those variants with an allele frequency $<20 \%$. Of note, 10 out of 14 patients with a TP53 lesion could be reported herein only by the use of next-generation sequencing. Most of ATM mutations were detectable by conventional PCR technique, but the extent and lack of hotspots in this gene makes capillary Sanger sequencing as time-consuming and labor-intensive as to preclude its use in the routine praxis. These two aspects strongly favor the use of targeted sequencing in routine CLL workout.

Surprisingly, $\beta 2$-microglobulin levels, associated for the first time to CLL 25 years ago, ${ }^{43}$ showed its predictive value even compared with the newer biological markers of intrinsic cell phenotypic and genomic features. Of note, ours is not the first study to observe this strong role of $\beta 2$-microglobulin in predicting outcome in this particular early-stage CLL subset. ${ }^{23,44}$ The reason why a significant proportion of patients show high $\beta 2$-microglobuline levels in the context of a low CLL burden disease still remains unclear. A positive expression of CD38 correlated with a shorter TTFT independently, a more expected finding, given that it showed an independent prognostic value (which ZAP70 and FISH findings failed to reach) in one of the largest study of prognostic markers carried out in Binet stage A patients with more than a thousand patients an 8 years of median follow-up. ${ }^{42}$

We acknowledge some caveats in our study. Considering FISH data as a loss of heterozygosity measure for VAF adjusting, can lead to underestimation of VAF in those cases where $17 p$ or $11 q$ clones might be under the sensibility threshold established for that probe. It is true that VAF would change, and TP53 or ATM VAF estimates of $<10 \%$ should be taken cautiously. However, those mutations would remain defined as subclonal, as none of our TP53 or ATM-mutated patients had a CLL population of $<25 \%$. In addition, it could be argued we did not sequenced all ATM exons, and that we are underestimating ATM mutational status. Certainly, though we tried to design a cost-effective amplicon panel, and it covers most of ATM mutations reported in CLL, only an ATM whole-exome sequencing study will address the precise clinical impact of these gene variants. Finally, the lack of clinical grade availability of deep-sequencing data precludes its use presently. The implementation will need to go through standardization of methods and validation of prognostic value in clinical trials.

In sum, our finding of an independent prognostic value of TP53 mutations, not detectable by conventional techniques in a subset of patients lacking treatment indication at baseline, adds another cobblestone to the positioning of amplicon deep-sequencing assays in established CLL diagnostics algorithms. The highthroughput determination of TP53 status, particularly in this set of patients frequently lacking high-risk chromosomal aberrations, emerges as a key step, not only for prediction modeling, but also for exploring mutation-specific therapeutic approaches and minimal residual disease monitoring.

\section{CONFLICT OF INTEREST}

The authors declare no conflict of interest.

\section{ACKNOWLEDGEMENTS}

This study was supported by a grant from Instituto de Salud Carlos III (PI13/02099) with participation of funds from FEDER (European Union).

\section{REFERENCES}

1 Abrisqueta P, Pereira A, Rozman C, Aymerich M, Gine E, Moreno C et al. Improving survival in patients with chronic lymphocytic leukemia (1980-2008): the Hospital Clinic of Barcelona experience. Blood 2009; 114: 2044-2050.

2 Rawstron AC, Bottcher S, Letestu R, Villamor N, Fazi C, Kartsios H et al. Improving efficiency and sensitivity: European Research Initiative in CLL (ERIC) update on the international harmonised approach for flow cytometric residual disease monitoring in CLL. Leukemia 2013; 27: 142-149.

3 van den Broek EC, Kater AP, van de Schans SA, Karim-Kos HE, Janssen-Heijnen ML, Peters WG et al. Chronic lymphocytic leukaemia in the Netherlands: trends in incidence, treatment and survival, 1989-2008. Eur J Cancer 2012; 48: 889-895.

4 Wierda WG, O'Brien S, Wang X, Faderl S, Ferrajoli A, Do KA et al. Multivariable model for time to first treatment in patients with chronic lymphocytic leukemia. J Clin Oncol 2011; 29: 4088-4095.

5 Molica S, Giannarelli D, Gentile M, Cutrona G, Di Renzo N, Di Raimondo F et al. The utility of two prognostic models for predicting time to first treatment in early chronic lymphocytic leukemia patients: results of a comparative analysis. Leuk Res 2013; 37: 943-947.

6 Tomlinson IP, Novelli MR, Bodmer WF. The mutation rate and cancer. Proc NatI Acad Sci USA 1996; 93: 14800-14803.

7 Landau DA, Carter SL, Getz G, Wu CJ. Clonal evolution in hematological malignancies and therapeutic implications. Leukemia 2014; 28: 34-43.

8 Dohner $\mathrm{H}$, Stilgenbauer $\mathrm{S}$, Benner $\mathrm{A}$, Leupolt $\mathrm{E}$, Krober $\mathrm{A}$, Bullinger $\mathrm{L}$ et al. Genomic aberrations and survival in chronic lymphocytic leukemia. N Engl J Med 2000; 343: 1910-1916.

9 Landau DA, Carter SL, Stojanov P, McKenna A, Stevenson K, Lawrence MS et al. Evolution and impact of subclonal mutations in chronic lymphocytic leukemia. Cell 2013; 152: 714-726.

10 Puente XS, Pinyol M, Quesada V, Conde L, Ordonez GR, Villamor N et al. Whole-genome sequencing identifies recurrent mutations in chronic lymphocytic leukaemia. Nature 2011; 475: 101-105.

11 Quesada V, Ramsay AJ, Lopez-Otin C. Chronic lymphocytic leukemia with SF3B1 mutation. N Engl J Med 2012; 366: 2530.

12 Wang L, Lawrence MS, Wan Y, Stojanov P, Sougnez C, Stevenson K et al. SF3B1 and other novel cancer genes in chronic lymphocytic leukemia. $N$ Engl J Med 2011; 365: 2497-2506

13 Rossi D, Khiabanian H, Spina V, Ciardullo C, Bruscaggin A, Fama R et al. Clinical impact of small TP53 mutated subclones in chronic lymphocytic leukemia. Blood 2014; 123: 2139-2147

14 Austen B, Powell JE, Alvi A, Edwards I, Hooper L, Starczynski J et al. Mutations in the ATM gene lead to impaired overall and treatment-free survival that is independent of IGVH mutation status in patients with B-CLL. Blood 2005; 106: 3175-3182.

15 Rossi D, Bruscaggin A, Spina V, Rasi S, Khiabanian H, Messina M et al. Mutations of the SF3B1 splicing factor in chronic lymphocytic leukemia: association with progression and fludarabine-refractoriness. Blood 2011; 118: 6904-6908.

16 Baliakas P, Hadzidimitriou A, Sutton LA, Rossi D, Minga E, Villamor $\mathrm{N}$ et al. Recurrent mutations refine prognosis in chronic lymphocytic leukemia. Leukemia 2015; 29: 329-336.

17 Hallek M, Cheson BD, Catovsky D, Caligaris-Cappio F, Dighiero G, Dohner $\mathrm{H}$ et al. Guidelines for the diagnosis and treatment of chronic lymphocytic leukemia: a report from the International Workshop on Chronic Lymphocytic Leukemia 
updating the National Cancer Institute-Working Group 1996 guidelines. Blood 2008; 111: 5446-5456.

18 Dohner H, Stilgenbauer S, Fischer K, Bentz M, Lichter P. Cytogenetic and molecular cytogenetic analysis of B cell chronic lymphocytic leukemia: specific chromosome aberrations identify prognostic subgroups of patients and point to loci of candidate genes. Leukemia 1997; 11: S19-S24.

19 Capello D, Guarini A, Berra E, Mauro FR, Rossi D, Ghia E et al. Evidence of biased immunoglobulin variable gene usage in highly stable B-cell chronic lymphocytic leukemia. Leukemia 2004; 18: 1941-1947.

20 Marasca R, Vaccari P, Luppi M, Zucchini P, Castelli I, Barozzi P et al. Immunoglobulin gene mutations and frequent use of $\mathrm{VH} 1-69$ and $\mathrm{VH} 4-34$ segments in hepatitis $C$ virus-positive and hepatitis $C$ virus-negative nodal marginal zone B-cell lymphoma. Am J Pathol 2001; 159: 253-261.

21 Giudicelli V, Chaume D, Lefranc MP. IMGT/V-QUEST, an integrated software program for immunoglobulin and T cell receptor V-J and V-D-J rearrangement analysis. Nucleic Acids Res 2004; 32: W435-W440.

22 Damle RN, Wasil T, Fais F, Ghiotto F, Valetto A, Allen SL et al. Ig V gene mutation status and CD38 expression as novel prognostic indicators in chronic lymphocytic leukemia. Blood 1999; 94: 1840-1847.

23 Gentile M, Cutrona G, Neri A, Molica S, Ferrarini M, Morabito F. Predictive value of beta2-microglobulin (beta2-m) levels in chronic lymphocytic leukemia since Binet A stages. Haematologica 2009; 94: 887-888.

24 Molica S, Giannarelli D, Levato L, Mirabelli R, Gentile M, Lentini M et al. A prognostic algorithm including a modified version of MD Anderson Cancer Center (MDACC) score predicts time to first treatment of patients with clinica monoclonal lymphocytosis (CMBL)/Rai stage 0 chronic lymphocytic leukemia (CLL). Int J Hematol 2014; 100: 290-295.

25 Shanafelt TD, Kay NE, Jenkins G, Call TG, Zent CS, Jelinek DF et al. B-cell count and survival: differentiating chronic lymphocytic leukemia from monoclonal B-cell lymphocytosis based on clinical outcome. Blood 2009; 113: 4188-4196.

26 Call TG, Norman AD, Hanson CA, Achenbach SJ, Kay NE, Zent CS et al. Incidence of chronic lymphocytic leukemia and high-count monoclonal B-cell lymphocytosis using the 2008 guidelines. Cancer 2014; 120: 2000-2005.

27 Molica S, Giannarelli D, Mirabelli R, Levato L, Gentile M, Lentini M et al. Changes in the incidence, pattern of presentation and clinical outcome of early chronic lymphocytic leukemia patients using the 2008 International Workshop on CLL guidelines. Exp Rev Hematol 2014; 7: 691-695.

28 Hallek M, Fischer K, Fingerle-Rowson G, Fink AM, Busch R, Mayer J et al. Addition of rituximab to fludarabine and cyclophosphamide in patients with chronic lymphocytic leukaemia: a randomised, open-label, phase 3 trial. Lancet 2010; 376 : $1164-1174$

29 Catovsky D, Richards S, Matutes E, Oscier D, Dyer MJ, Bezares RF et al. Assessment of fludarabine plus cyclophosphamide for patients with chronic lymphocytic leukaemia (the LRF CLL4 Trial): a randomised controlled trial. Lancet 2007; 370 230-239.

30 Strati P, Keating MJ, O'Brien SM, Ferrajoli A, Burger J, Faderl S et al. Outcomes of first-line treatment for chronic lymphocytic leukemia with $17 p$ deletion. Haematologica 2014; 99: 1350-1355.

31 Farooqui MZ, Valdez J, Martyr S, Aue G, Saba N, Niemann CU et al. Ibrutinib for previously untreated and relapsed or refractory chronic lymphocytic leukaemia with TP53 aberrations: a phase 2, single-arm trial. Lancet Oncol 2015; 16: 169-176.

32 Malcikova J, Stano-Kozubik K, Tichy B, Kantorova B, Pavlova S, Tom N et al. Detailed analysis of therapy-driven clonal evolution of TP53 mutations in chronic lymphocytic leukemia. Leukemia 2015; 29: 877-885.
33 Damm F, Mylonas E, Cosson A, Yoshida K, Della Valle V, Mouly E et al. Acquired initiating mutations in early hematopoietic cells of CLL patients. Cancer Discov 2014; 4: 1088-1101.

34 Jethwa A, Hullein J, Stolz T, Blume C, Sellner L, Jauch A et al. Targeted resequencing for analysis of clonal composition of recurrent gene mutations in chronic lymphocytic leukaemia. Br J Haematol 2013; 163: 496-500.

35 Cuneo A, Bigoni R, Rigolin GM, Roberti MG, Bardi A, Cavazzini F et al. Late appearance of the 11q22.3-23.1 deletion involving the ATM locus in B-cell chronic lymphocytic leukemia and related disorders. Clinico-biological significance. Haematologica 2002; 87: 44-51.

36 Austen B, Skowronska A, Baker C, Powell JE, Gardiner A, Oscier D et al. Mutation status of the residual ATM allele is an important determinant of the cellular response to chemotherapy and survival in patients with chronic lymphocytic leukemia containing an 11q deletion. J Clin Oncol 2007; 25: 5448-5457.

37 Agathanggelou A, Weston VJ, Perry T, Davies NJ, Skowronska A, Payne DT et al. Targeting the Ataxia Telangiectasia Mutated-null phenotype in chronic lymphocytic leukemia with pro-oxidants. Haematologica 2015; e-pub ahead of print 3 April 2015.

38 Weissmann S, Roller A, Jeromin S, Hernandez M, Abaigar M, Hernandez-Rivas JM et al. Prognostic impact and landscape of NOTCH1 mutations in chronic lymphocytic leukemia (CLL): a study on 852 patients. Leukemia 2013; 27: 2393-2396.

39 Mansouri L, Cahill N, Gunnarsson R, Smedby KE, Tjonnfjord E, Hjalgrim H et al. NOTCH1 and SF3B1 mutations can be added to the hierarchical prognostic classification in chronic lymphocytic leukemia. Leukemia 2013; 27: 512-514.

40 Schnaiter A, Paschka P, Rossi M, Zenz T, Buhler A, Winkler D et al. NOTCH1, SF3B1, and TP53 mutations in fludarabine-refractory CLL patients treated with alemtuzumab: results from the CLL2H trial of the GCLLSG. Blood 2013; 122: 1266-1270.

41 Balatti V, Bottoni A, Palamarchuk A, Alder H, Rassenti LZ, Kipps TJ et al. NOTCH1 mutations in CLL associated with trisomy 12. Blood 2012; 119: 329-331.

42 Pepper C, Majid A, Lin TT, Hewamana S, Pratt G, Walewska R et al. Defining the prognosis of early stage chronic lymphocytic leukaemia patients. $\mathrm{Br} J$ Haematol 2012; 156: 499-507.

43 Simonsson B, Wibell L, Nilsson K. Beta 2-microglobulin in chronic lymphocytic leukaemia. Scand J Haematol 1980; 24: 174-180.

44 Oliveira AC, Fernandez de Sevilla A, Domingo A, De La Banda E, Domingo-Domenech E, Mercadal S et al. Prospective study of prognostic factors in asymptomatic patients with B-cell chronic lymphocytic leukemia-like lymphocytosis: the cut-off of $11 \times 10(9) / L$ monoclonal lymphocytes better identifies subgroups with different outcomes. Ann Hematol 2015; 94: 627-632.

45 Rossi D, Fangazio M, Rasi S, Vaisitti T, Monti S, Cresta S et al. Disruption of BIRC3 associates with fludarabine chemorefractoriness in TP53 wild-type chronic lymphocytic leukemia. Blood 2012; 119: 2854-2862.

46 Jebaraj BM, Kienle D, Buhler A, Winkler D, Dohner H, Stilgenbauer S et al. BRAF mutations in chronic lymphocytic leukemia. Leuk Lymphoma 2013; 54 1177-1182.

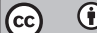

This work is licensed under a Creative Commons Attribution 4.0 International License. The images or other third party material in this article are included in the article's Creative Commons license, unless indicated otherwise in the credit line; if the material is not included under the Creative Commons license, users will need to obtain permission from the license holder to reproduce the material. To view a copy of this license, visit http://creativecommons.org/licenses/ by/4.0/

Supplementary Information accompanies this paper on Blood Cancer Journal website (http://www.nature.com/bcj) 